\title{
Investigation of Dimethyl Ether Combustion Instabilities in a Partially - Premixed Gas Turbine Model Combustor Using High-Speed Laser Diagnostics
}

\author{
Patton M. Allison ${ }^{*}$ Y YunTao Chen ${ }^{\dagger}$, and James F. Driscoll ${ }^{\ddagger}$ \\ Department of Aerospace Engineering, University of Michigan, Ann Arbor MI 48109 ${ }^{1}$
}

\begin{abstract}
Combustion instabilities in gas turbine engines often give rise to acoustic resonances. These resonances occur as manifestations of different acoustic modes, of which a single or multiple modes may be present. In this work, the acoustic behavior of a gas turbine model combustor, developed at DLR Stuttgart by W. Meier et al., was investigated using dimethyl ether (DME). The equivalence ratio and air mass flow rate were systematically varied. The results did not correspond to any one instability mechanism. It is concluded that, in the current burner configuration, integrated-acoustics occur that involve a combination of mechanisms, including a Helmholtz-type resonance from the plenum and convective-acoustic effects. To understand the instability, accurate measurements are needed of the correlation between heat release rate fluctuations and pressure fluctuations. Thus heat release rate must be recorded as a function of time and space. However conventional chemiluminescence offers only a line-of-sight measurement. High-speed formaldehyde planar laser-induced fluorescence was applied to study the motion of flame surfaces in response to the pressure oscillations of the instability. Flame shape has been correlated with instability strength and presence. The flame surface density and surface area fluctuated at the acoustic frequency and displayed motions correlated with the precessing vortex core (PVC) rotation. In nonresonating flames, the behavior of the formaldehyde structure and marked flame surfaces were dominated by the PVC motion, but the degree of surface area fluctuations was reduced compared to unstable flames. Results show that the frequency of the combustion instability varies with several operational conditions, including gas velocity, equivalence ratio, and convective time delays.
\end{abstract}

\section{Introduction}

$\mathrm{F}$ LAME stabilization and anchoring, in gas turbine burners, are achieved with swirling flow which leads to the creation of a precessing vortex core (PVC) and enhanced mixing. The addition of swirl to a combustor has been shown to allow stable operation over a range of conditions and regimes ${ }^{1}$. However, they are also subject to thermoacoustic oscillations that can lead to acoustic instabilities. These instabilities arise when there is proper coupling between the pressure field and heat release field to satisfy the Rayleigh criterion ${ }^{2}$, such that they are in phase. These pressure fluctuations may also induce fluctuations in the oxidizer and fuel flow rates such that equivalence ratio oscillations occur ${ }^{3}$. These equivalence ratio oscillations continue to feed the flame causing cyclical variations in heat release and pressure. Figure 1 demonstrates some of the mechanisms involved in inducing instabilities in swirl flames. This paper focuses on a dual-swirl burner that undergoes a natural combustion instability at a frequency in the range between 250 to $480 \mathrm{~Hz}$. The naturally occurring combustion instability has previously been shown to be the product a Helmholtz and a convective-acoustic mechanism which also displays a flame speed dependence ${ }^{4}$. This instability introduces oscillations in fuel and air mass flow rates which affects mixing, stabilization, instability strength, flame shape, and anchoring. These combustion parameters have been investigated by studying the interaction between the flame and fuel-air mixing for varying fueling conditions in methane, propane, ethylene, and syngas flames ${ }^{4}$. This current work focuses on identifying similar interactions in dimethyl ether flames with the addition of high-speed laser diagnostics to evaluate flame surface density measurements and preheat zone marking.

\footnotetext{
*Graduate Student Research Assistant. Member, AIAA, pallison@umich.edu

${ }^{\dagger}$ Graduate Student Research Assistant. Member, AIAA, yuntaoc@umich.edu

${ }^{\ddagger}$ Professor of Aerospace Engineering, University of Michigan, Fellow, AIAA, jamesfd@umich.edu
} 
The swirl burner, known as the gas turbine model combustor (GTMC), was developed by Meier and colleagues at DLR Stuttgart. It has the advantage of having a canonical axisymmetric swirler-design yet still exhibiting the fundamental physics associated with gas turbine flames. It contains two swirling air streams, which surround an annular fuel stream. Comprehensive measurements have been conducted in the burner-facility at DLR ${ }^{5,6}$ to investigate flame-structure, flame-dynamics, and flow-field structures for a few selected methane-fueled conditions. In present study, the change of behavior for these components when fuel is switched to DME is studied. These components will be evaluated by studying high-speed formaldehyde PLIF videos of flame motion and shape, and pressure measurements of acoustic resonances. Flame lift-off height oscillations can be induced by variations in the mixing as well as air flow rate distribution. It has been observed in the GTMC that air flow redistribution can occur between the two swirlers, which are fed from a common plenum ${ }^{7,8}$. Dynamic swirler air flow mass flow rate variations can result in large redistributions of heat release which can affect the flame shape and pressure field.

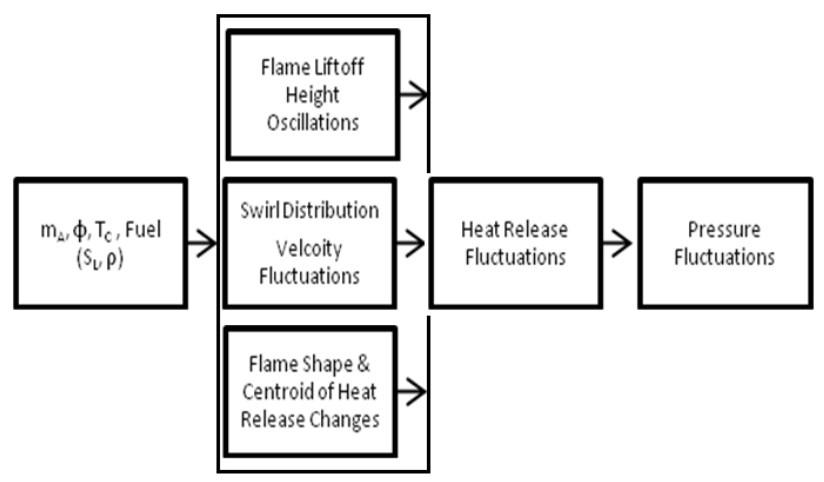

Figure 1. Proposed system of thermoacoustic relationships.

Another aspect of this study is to investigate the behavior of DME in highly turbulent flames such as in the GTMC burner. The chemical properties and reaction parameters of DME under laminar flame conditions have been studied by many researchers. Andersson et al. ${ }^{9}$ used laser-based combustion diagnostics methods and determined the optical properties of DME, such as its flame emission spectrum and Raman cross-section area. Fisher, Dryer, and Curran investigated the reaction kinetics of DME with flow reactors at both high temperature (1000K to $1100 \mathrm{~K})^{10}$ and low temperature $(550 \mathrm{~K} \text { to } 850 \mathrm{~K})^{11}$. Mcilroy et al. ${ }^{12}$ utilized a molecular beam mass spectrometer (MBMS) and laser diagnostics to examine the temperature and species profiles in a low pressure $\mathrm{DME} / \mathrm{O}_{2} / \mathrm{Ar}$ flame. Kaiser et al. ${ }^{13}$ examined the species and temperature profiles of DME/Air flame at ambient condition over an open flat flame burner with gas chromatography and Fourier transform infrared (FTIR) spectroscopy. The laminar burning velocities for a premixed DME/Air mixture have also been investigated at ambient conditions by Zhao et al. ${ }^{14}$ and at elevated pressure (up to $10 \mathrm{~atm}$ ) by Qin et al. ${ }^{15}$ More recently, Brackmann et al. ${ }^{16}$ employed a series of laser diagnostics methods, including coherent anti-Stokes Raman Spectroscopy (CARS), Rayleigh scattering, and laserinduced fluorescence (LIF) to quantitatively explore the species and temperature distributions in a counter-flow burner. Wang et al. ${ }^{17}$ used particle image velocimetry (PIV) and laser Doppler velocimetry (LDV) to examine the propagation speed and extinction strain rate of a premixed DME/Air flame. All of the aforementioned studies have quite comprehensively investigated the various properties of DME flames, albeit only under laminar conditions. The efforts to determine the behavior of DME flames in turbulent regimes have just been started very recently. The only studies that are known to the authors are those conducted by Fuest et al. ${ }^{18}$ and Gabet et al. ${ }^{19}$ Both of these studies were done on turbulent jet configurations and analyzed with LIF and laser scattering techniques.

The new aspects of the present work are the following. High-speed formaldehyde PLIF was conducted at 4 $\mathrm{kHz}$ to investigate the time-resolved motions of the flame surface relative to the fluctuating pressure field in reacting cases. As well, frequencies of the flame oscillations that correspond to the frequency of the rotation of the vortex core are noted. The flame surface densities for resonant and non-resonant cases are compared to understand the importance of the spatial distribution of flame surface area. Flame geometry and shape is seen to have a major effect on instability presence and strength. This paper reports that there are significant differences between the spatial and temporal flame shape for resonant and non-resonant flames. It is also shown that flame geometry can be more accurately determined from high-speed PLIF measurements than from conventional chemiluminescence alone. 
While chemiluminescence images can be taken at high-speed, the collected data represents line-of-sight integrated information about the flame. Spatial asymmetries will be averaged out due to this integration and timeresolved motions will appear to be symmetric. Most importantly though, is the ability to distinguish differences in increased heat release. The local heat release can be altered by fluctuations in flame surface area or changes to the local reaction rate. Chemiluminescence videos cannot distinguish between these two causes and can only report the total effect of increased heat release. However, high-speed PLIF measurements can capture the independent effect of changes in flame surface area.

Hence, the uniqueness of our present study lies in the combination of a complex geometry swirl burner under highly turbulent operating conditions with the relatively new and very promising fuel of DME. The results will improve our understanding of the differences between DME flames and other similar fuels, and especially its influence on acoustic instabilities.

\section{Experimental Facility and Operation}

A gas turbine model combustor (GTMC) as seen in Fig. $2^{6}$ is used to study acoustic behavior and performance. The injector consists of a central air nozzle, an annular fuel nozzle, and a co-annular air nozzle. Both air nozzles supply swirling dry air at atmospheric pressure and temperature from a common plenum. The inner air nozzle has an outer diameter of $15 \mathrm{~mm}$ and the annular nozzle has an inner diameter of $17 \mathrm{~mm}$ and an outer diameter of $25 \mathrm{~mm}$. The measured swirl number is approximately 0.55 . Non-swirling fuel is provided through three exterior ports fed through the annular nozzle which is subdivided into $720.5 \mathrm{~mm}$ x $0.5 \mathrm{~mm}$ channels. The width of the fuel annulus is less than $0.5 \mathrm{~mm}$. The exit plane of the central air nozzle and fuel nozzle lies $4.5 \mathrm{~mm}$ below the exit plane of the outer air annulus. The exit plane of the outer air annulus will be referred to as the burner face. The combustion chamber has a square cross section of $85 \mathrm{~mm}$ in width and $110 \mathrm{~mm}$ in height. The exit of the burner has a tapered lid which leads to an exhaust chimney with a diameter of $40 \mathrm{~mm}$ and a height of $50 \mathrm{~mm}$. The burner is operated with 4 fused silica windows, with a thickness of $1.5 \mathrm{~mm}$, for flame visualization. The burner was fired dimethyl ether fuel. An external cylindrical chamber was used for the equal division of the fuel flow into three separate lines which lead to the fuel ports on the burner.

Mass flow rates for the air and individual fuel lines leading to the burner were controlled by sonically choked orifices. Table 1 lists the operating parameters investigated. Of note is the fueling case primarily studied by Weigand et al. ${ }^{6}$ identified as Case B. This methane case has an air mass flow rate of $282 \mathrm{~g} / \mathrm{min}$ and an equivalence ratio of 0.75 . The fueling conditions for Case $B$ have been the standard for which these measurements have been based on. The air mass flow rate and equivalence ratio for DME have been varied in a range around the Case B operating condition. A PCB piezoelectric transducer was mounted in the plenum, which has a diameter of $80 \mathrm{~mm}$ and a length of $72 \mathrm{~mm}$. For a given operating condition, pressure measurements are taken at a set burner reference temperature. The frequency and amplitude in the plenum was previously shown to be not significantly different from that in the combustion chamber, with the exception of a phase shift ${ }^{20,21}$. The reported frequencies are accurate to $\pm 0.5 \%$ and the calculated amplitude is accurate to $\pm 3 \%$. For a given operating condition, pressure measurements are taken at a set burner reference temperature for 5 seconds at $20 \mathrm{kHz}$, yielding 100,000 data points. High-speed videos of the total flame chemiluminescence were taken using a Phantom V711 high-speed camera. Videos were taken at a frame rate of $4000 \mathrm{fps}$ with an exposure of $80 \mu$ s at 1280 x 800 pixels. The resolution of each of the images is 138 $\mu \mathrm{m} /$ pixel. 


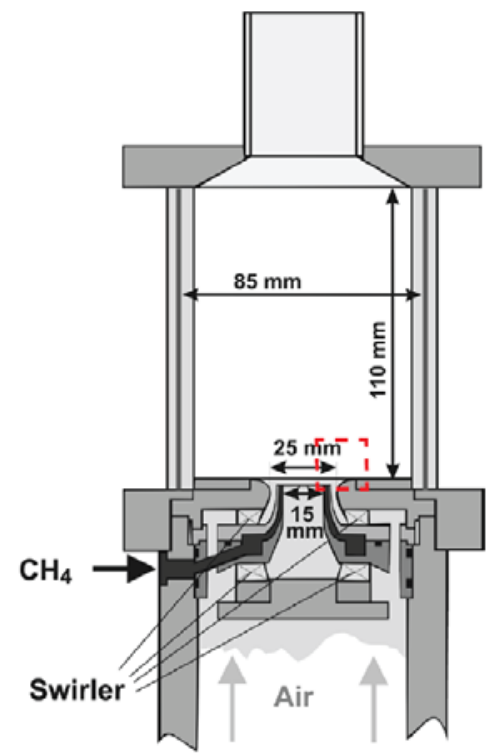

Figure 2. GTMC schematic drawing ${ }^{6}$ depicting air and fuel flow paths, as well as swirl vane locations. The red dotted box indicates the field of view of the formaldehyde PLIF measurements.

Table 1. Fueling Parameters Investigated

\begin{tabular}{ccc}
\hline Fuel & $\mathbf{m}_{\mathbf{a}}, \mathbf{g} / \mathbf{m i n}$ & $\boldsymbol{\Phi}_{\text {Global }}$ \\
\hline Dimethyl Ether & $140-353$ & $0.65-1.2$ \\
\hline
\end{tabular}

In this study the $4_{0}^{1}$ transition in formaldehyde is excited using a frequency-tripled diode pumped Nd:YAG Quantronix Hawk HP laser at $355 \mathrm{~nm}$. At $4 \mathrm{kHz}$, the laser energy per pulse was $9.7 \mathrm{~mJ}$ and the sheet height was 25 $\mathrm{mm}$. Fluorescence was recorded with LaVision HS-IRO intensifier with a gate time of $200 \mathrm{~ns}$, a Phantom v711 camera, and a $105 \mathrm{~mm}$ Nikon micro-Nikkor f/2.8 lens. Spatial resolution was $76 \mu \mathrm{m}$ per pixel. A GG-385 filter removed laser interference below $385 \mathrm{~nm}$. After background corrections and filtering, the signal to noise ratios varied between 6.5-11.2.

\section{Results and Discussion}

\section{A. DME Acoustic Characterization}

Pressure measurements of DME flames at the Case B air flow rate, $282 \mathrm{~g} / \mathrm{min}$, were taken at various equivalence ratios and air mass flow rates. Figure 3 depicts this variation alongside the acoustic trends of other fuels previously studied in the GTMC. The DME trend is similar to propane in behavior until the flames become fuel rich, at which point the behavior is monotonic and similar to ethylene.

Instabilities that are of a convective-acoustic type display a frequency that varies with the gas velocity, while those that are pure organ tones or Helmholtz types do not. To investigate these effects the air mass flow rate was varied. Figure 4 indicates that the frequency of the instability increases with an increase in the mass flow rate. This suggests that the instability depends on a Strouhal number and has a convective-acoustic component. DME generally shows increasing resonant frequency with flow rate as prescribed by a convective acoustic effect. 


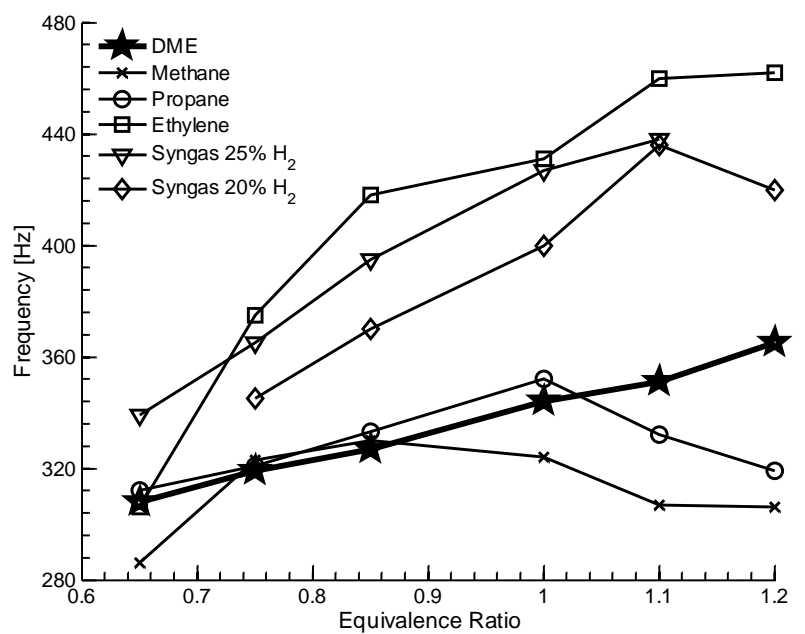

Figure 3. Frequency as a function of equivalence ratio for various fuels, including DME.

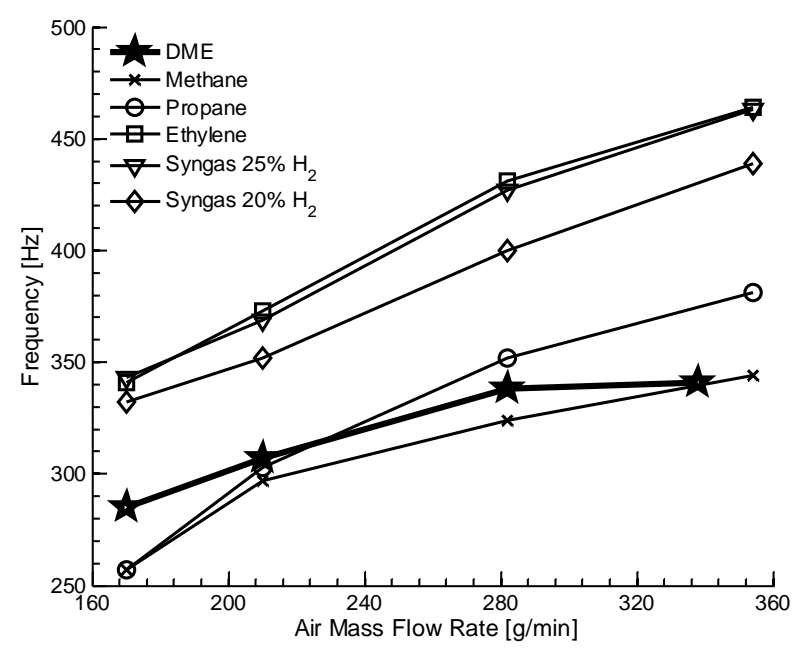

Figure 4. Frequency as a function of air mass flow rate for various fuels, including DME.

Pressure measurements were taken simultaneously with high-speed videos of the line of sight chemiluminescence. These measurements showed detailed flame motions occurring over a given acoustic cycle. For the chemiluminescence images, information regarding the average flame shape, liftoff height, average liftoff fluctuation distance, and frequency of heat release could be investigated. When combined with pressure data, Rayleigh indices $^{22}$ can be calculated which determine the degree of coupling between the pressure field and heat release.

Figure 5 shows that there are noticeable differences in the flame shapes between the resonating case and the nonresonating case, as determined by time-averaged chemiluminescence. It was previously determined from LDV velocity measurements that the flame shape is set by the distribution of air flow rate through the two swirler ${ }^{21}$. Resonating flames exhibited a larger measured radial velocity at the exit of the nozzle due to higher flow through the outer swirler. Higher radial velocities are essential to the establishment of flat flames. When the flame was nonresonant, low radial flow rates were observed and the flame had a V-shape. 


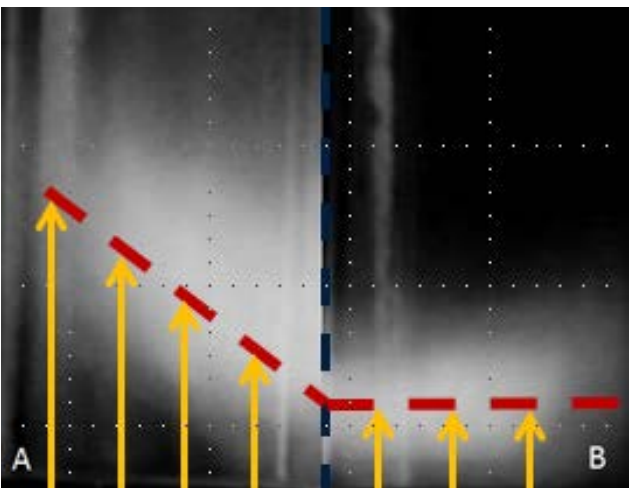

Figure 5. Time-averaged chemiluminescence of V-shaped, non-resonant flames (a) and flat, resonating flames(b).

Figure 4 is evidence that a convective-acoustic mechanism is present because the frequency of the instability is found to scale with the gas velocity. This mechanism often is associated with equivalence ratio oscillations. Inhomogeneities in fuel-air ratio can create packets of fluid that are convected and lead to unsteady heat release. Pressure fluctuations that are reflected back to the injector provide the feedback that triggers the formation of the next packet. The frequency of the feedback is set by the characteristic time of the cycle for packets to be convected to the flame and then have pressure waves reflected back. This characteristic time is reduced if convection speeds are increased, thus increasing the frequency of the instability, as seen in Fig. 4.

The spatial location of the flame alters the convective time scales and thus determines the degree to which the instability is excited. The convective-acoustic mode is controlled by two timescales, a convective timescale for perturbations to reach the flame and an acoustic time scale for perturbations to return to the nozzle. The instability frequency is inverse to the sum of these scales, as given in Equation 1. For relatively flat flames, this convective time scale is uniform across the flame surface. However, for V-shaped flames, the distance to the flame varies over the length of the surface, and thus the time scale also varies, as seen by the arrows in Figure 5. Thus in flat flames, if the phase difference is appropriate, the entire flame contributes to the amplification. However, in V-shapes flames, only a portion of the flame surface may have the correct phase difference to aid in amplification, and the resonance may not occur.

$$
\tau_{c}=\frac{l_{f}}{U}+\frac{\delta_{f}}{S_{L}}+\frac{l_{f}}{c}
$$

Figure 6 represents flame motions over the course of a liftoff/flashback cycle for a DME flame operated at Case B conditions. The flame lifts off and then returns to a flat shape. These regions will be further analyzed with formaldehyde PLIF measurements. As the flame moves back upstream to the injector, this region remains unburned. In Scene 3, there is still some unburned space in this region until the flame fully propagates back to the burner face in Scene 4. The cycle repeats with the flame then lifting off again. Similar behavior is observed in alkane flames.

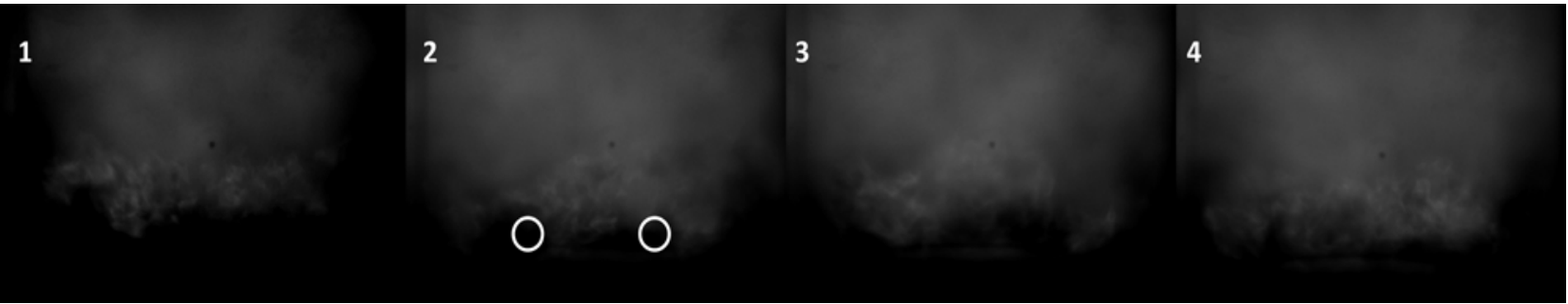

Figure 6 DME flame motion, conditions over a single acoustic cycle. The white circles represent the regions investigated with formaldehyde PLIF.

This liftoff and flashback cycling occurs at the same frequency as the pressure oscillations for all fuels examined. Chemiluminescence and pressure spectra are given Figure 7. For DME flames, the average liftoff height was $17 \mathrm{~mm}$. The flame motion can also be examined for periodic behavior, as given in Figure 8, which depicts the 
flamefront oscillating at the same frequencies as the total chemiluminescence and pressure signals. Propagation speeds for the flame motion were also calculated for the upstream and downstream motions. The upstream and downstream speeds were $2.5 \mathrm{~m} / \mathrm{s}$ and $1.9 \mathrm{~m} / \mathrm{s}$, respectively. The average propagation fluctuation for a DME flame was $0.52 \pm 1.2 \mathrm{~mm}$. These results indicate a high degree of motion and rapidly changing flame sizes and positions.

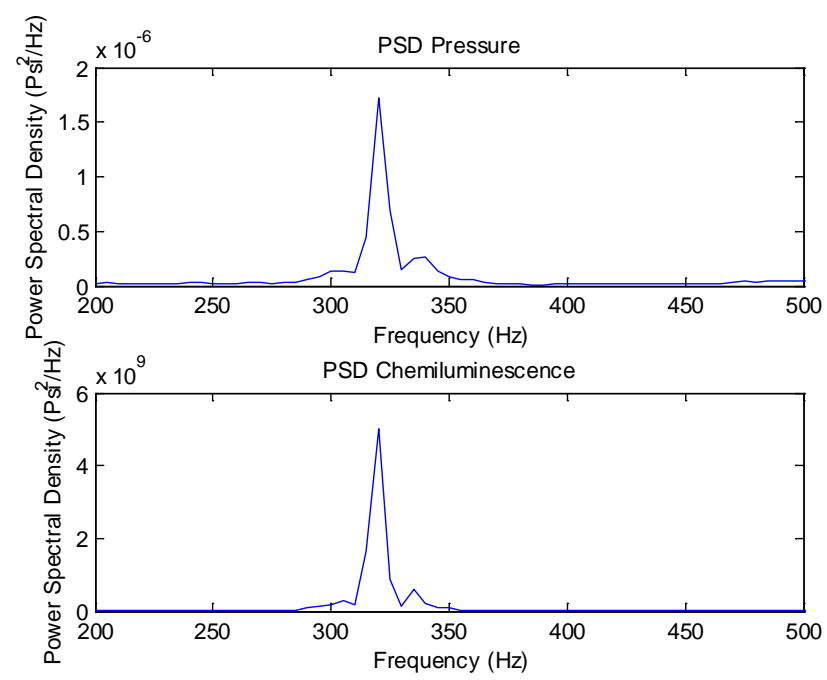

Figure 7. Pressure and chemiluminescence power spectral densities DME flames operated at Case B.

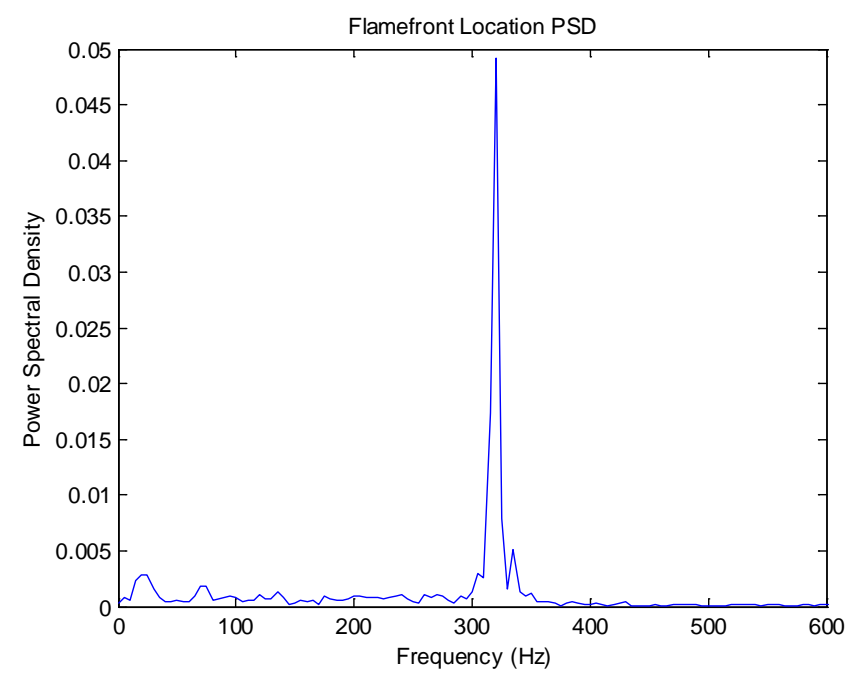

Figure 8. Flamefront power spectral densities DME flames operated at Case B.

For these Rayleigh indices, the pressure was recorded at a port located in the combustor wall, $1 \mathrm{~cm}$ above the injector face. Figure 9 depicts the Rayleigh index field of DME flames operated at an equivalence ratio of 0.85 and 1.0. In Figure 10, Rayleigh indices for previously studied fuels are included for comparison. Rayleigh indices greater than zero represent thermo-acoustically amplified flames where the pressure field and heat release are in phase, thus satisfying the Rayleigh criterion. Indices less than zero represent dampening. Figures 10a and 10b show Rayleigh index and average chemiluminescence for a syngas flame. The index field is populated by values that are close to zero or slightly negative which is indicative of a field with little coupling between the pressure and heat release. Figures 10c and 10e display the same characteristics for a resonating flame and there exists a strong region of amplification surrounded by dampened regions, similar to that shown by Kang et al. ${ }^{23}$ There are noticeable differences in the flame shapes between the resonating flames and the non-resonating flames. A non-resonating 
flame shows better anchoring and has an axially extended V-shape profile. While for a resonating flame, its profile is wider at its base than the non-resonating flame, which implies a higher degree of premixing. Flat flames are thermally compact and concentrate heat release in a smaller portion of the burner than an axially extended V-flame. Thus, V-flames are less effective at applying thermal energy toward acoustic amplification and satisfaction of the Rayleigh criterion. DME flames are interesting in that while they display flat flame features, the Rayleigh index as seen in Fig 9 only depicts a flame that is weakly resonating in comparison to other fuels operated at near the same fueling conditions.

a)

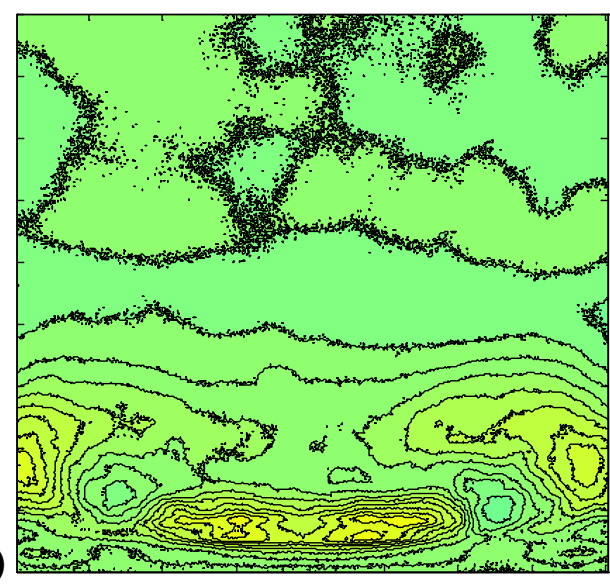

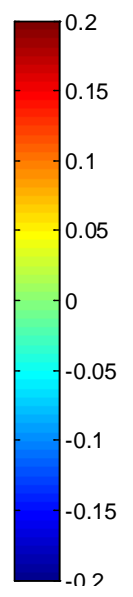

b)

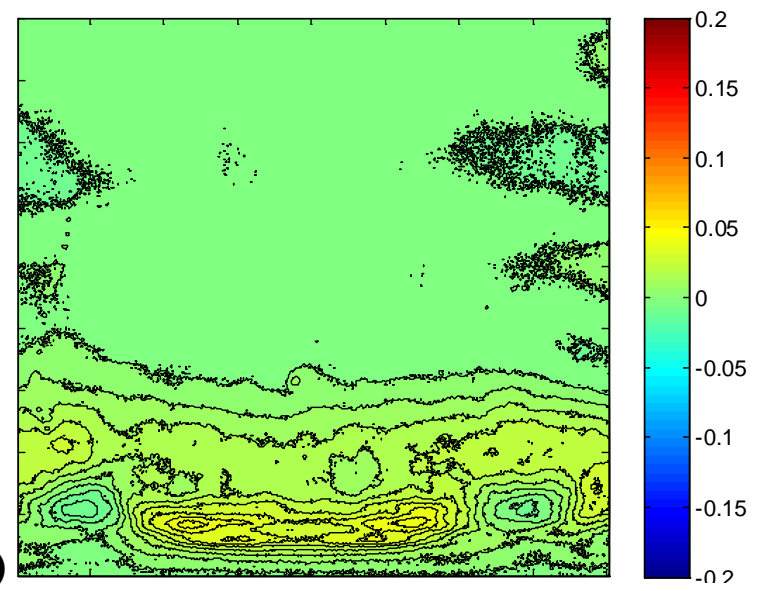

Figure 9. Rayleigh index of DME flames for a) $\varphi=0.85$ and $b) \varphi=1.0, \mathbf{m}_{a}=282 \mathrm{~g} / \mathrm{min}$.
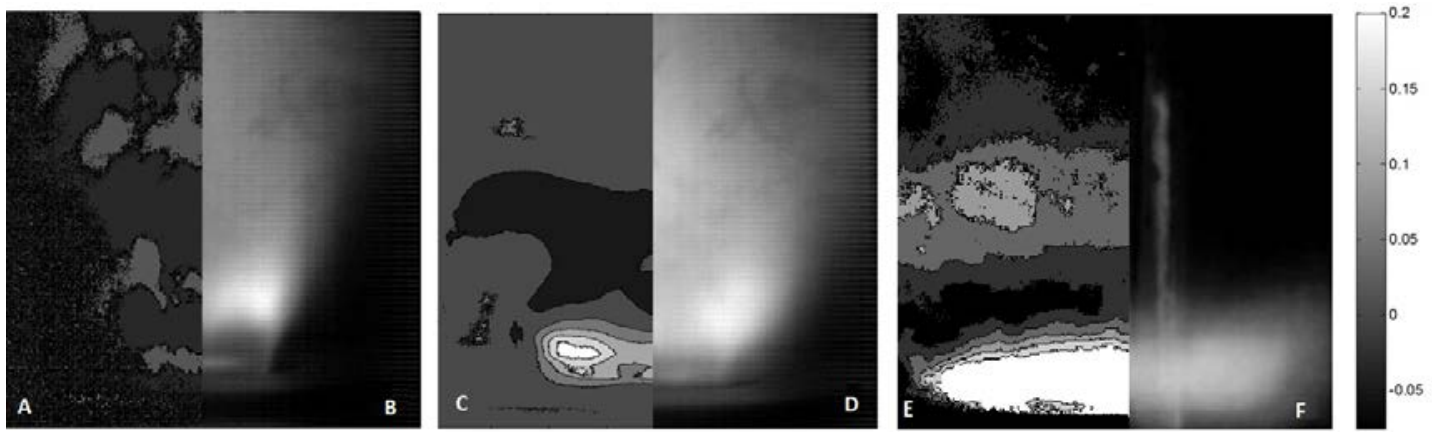

Figure 10. Rayleigh index and chemiluminescence of syngas and propane flames for $\varphi=1, m_{a}=282$ g/min. (a,b) Stable syngas flame, $40 \% \mathrm{H}_{2}, 60 \%$ CO. (c,d) Unstable resonating syngas flame, $25 \% \mathrm{H}_{2}$, $75 \%$ CO. (e,f) Unstable resonating propane flame.

\section{B. Flame Surface Density and Surface Area from kHz PLIF}

The flame surface density (FSD) here is used as an indicator of the local volumetric heat release rate (q'); this was done previously by Steinberg et al. ${ }^{21}$, and the two quantities were shown to be correlated in the DNS work of Tap et al. ${ }^{24}$. Also, subgrid reaction rate rate $\left(\omega_{\mathrm{sg}}\right)$, which is proportional to heat release rate, has been modeled to be proportional to $\rho_{\mathrm{R}} \cdot \mathrm{S}_{\mathrm{L}} \cdot \mathrm{FSD}{ }^{25,26}$. To mark the flame surface, the steep gradient on the flame side of the formaldehyde region is used, since it corresponds to the maximum production of $\mathrm{CH}$ radicals in the reaction zone ${ }^{19}$.

Flame surface was identified using edge marking and gradient thresh-holding. The high gradient edge where the flame exists is easily distinguished from low PLIF signal gradients that result from fuel decomposition. Figure 11a is a typical PLIF image that is then binarized to eliminate background noise. A canny edge tracking method marks the detected edges that are seen in Figure 11b. Next, the gradient is calculated along this edge using a central difference method. Gradients that exceed a threshold are defined to be flame surfaces, as seen as blue pixels in Fig. 11c, while 
red pixels denote the edges that did not pass the threshold requirement. Those below the threshold are considered markers of fuel decomposition into formaldehyde or noise in the signal causing false edges.

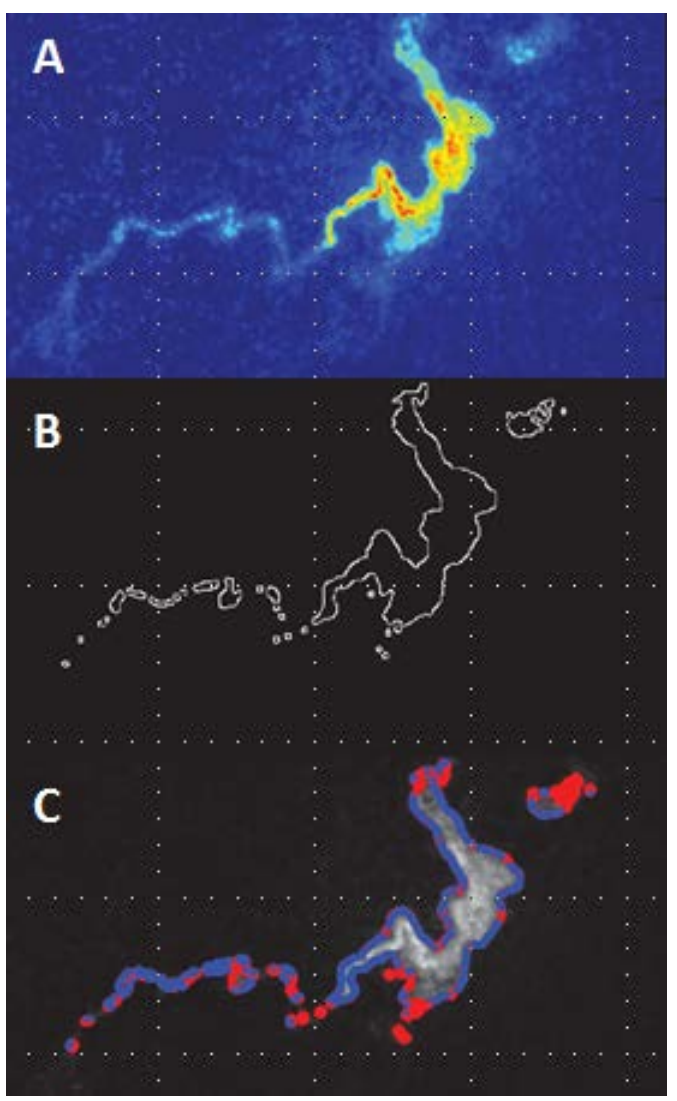

Figure 11. Detection method of flame surface segments from formaldehyde PLIF images. Raw image (a) and all detected edges (b). In (c), the blue edges are segments marked as flame surface and the red edges have been neglected by the gradient threshold.

Once the appropriate surfaces have been marked, the image can be reduced to a series of surface contours. This is essentially an image consisting of only surface edges. This is conducted for 4000 images in each case study, and frequency statistics can be applied to them. Since each pixel represents a physical segment of flame length, the pixel resolution of the image can be used to convert the edge segments into flame surface length. From this estimate, the total flame length in the laser sheet plane can be studied to see how it evolves in time. Finally, the flame surface density can be calculated by binning the image and measuring the number of flame surface contours that lie in each spatial bin.

Flame surface density is the average area of flame surface that lies within a 3-D volume in space. Given that the formaldehyde image measurements are 2-D in nature, the FSD can be estimated by:

$$
F S D_{x y}=\frac{\delta L_{f}}{\delta A_{x y}}
$$

where $\delta \mathrm{L}_{\mathrm{f}}$ is total flame length that lies inside the interrogation box area $\delta \mathrm{A}_{\mathrm{xy}}$ in 4000 images. Each box is $0.76 \mathrm{x}$ $0.76 \mathrm{~mm}$. Figure 12 depicts the measured FSD for a resonating and a non-resonating case. The width of the color contours indicates the width of the turbulent flame brush. For both cases, there is little flame surface density on the centerline, although the non-resonating case has more FSD at radially-inward locations, while for the resonating case the FSD is distributed radially further out. In both cases the FSD is confined within two lobes that are symmetric around the centerline. For the non-resonant case, each lobe is seen to be composed of two separate zones. Previous PIV measurements ${ }^{21}$ show that a shear layer exists at the location between these two zones. This layer separates the central and outer recirculation zones and may explain why each FSD lobe is divided into two regions in Fig. 12a. 

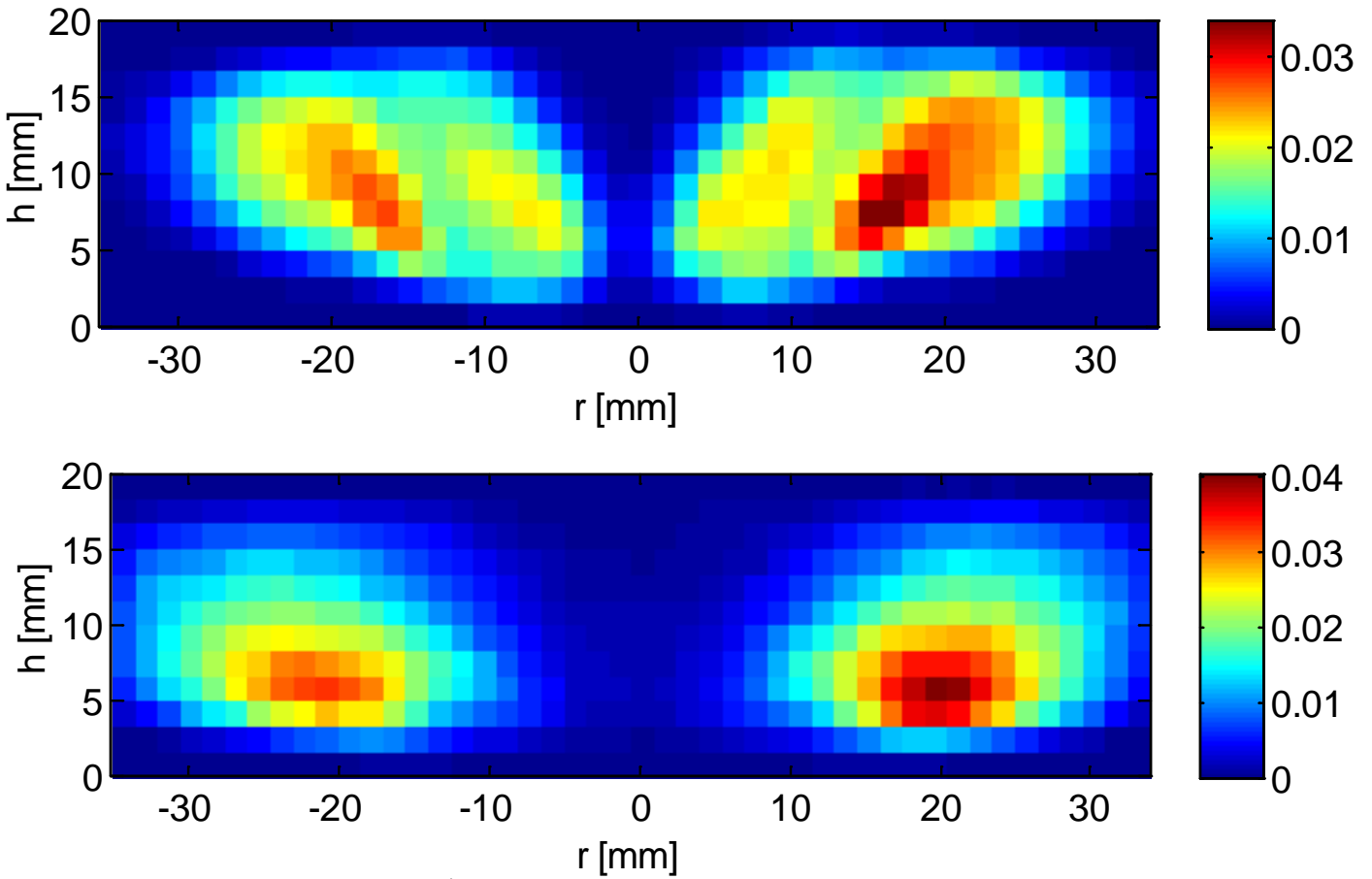

Figure 12. Flame surface density $\left(\mathrm{mm}^{-1}\right)$ for non-resonant (top) and resonant (bottom) DME flames.

Figure 12 shows that the highest probability of flame occurrence is at the outer edges of the brush. The nonresonating case displays an initial rise in flame surface marking the inner zone of each lobe, followed by a secondary rise marking the outer zone of the lobes. As well, the resonating case shows that the maximum wrinkling occurs at a larger radial distance. With higher turbulence intensities, it is expected that the flame would become more wrinkled and the total flame surface area would increase. This is not apparently clear from measurements of flame length, which give approximately the same average in-plane length for both cases. In fact the total integrated FSD for each case, results in roughly the same degree of flame surface. However a more important metric would be the 3D surface area of the flame, which assuming that the flame is axisymmetric, can be estimated by the convolution of the flame surface length with its radial position, resulting in a radially weighted surface area parameter, such that wider flames will have a larger area. This metric dictates that flame surface which is distributed further from the centerline contributes more to the total surface area of the flame. The flame area per unit length parameter $\Psi$ is defined to be:

$$
\Psi=\int_{-\infty}^{+\infty} r \cdot F S D d h
$$

$\Psi$ is proportional to flame area per unit distance $(\delta \mathrm{r})$ in the radial direction, which has been integrated in the streamwise direction, h. The radial profiles of $\Psi$ that are plotted in Fig. 13 indicate the radial location where flame surface density is maximum. This is consistent with the FSD contours of Fig. 12, but the $\Psi$ parameter is weighted by the radial coordinate (r) which appears in Eq. 3. Figure 13 suggests that a sufficiently large value of $\Psi$ (surface area per unit length in the radial direction) coincides with the presence of a combustion instability, which globally means that the flame brush is flat and thin. The non-resonating flame has a smaller profile of $\Psi$ because it is Vshaped. By having flame surface occur at large radial locations, as in flat flames, the convoluted flame surface area is greater, which is correlated with larger heat release. Coupled with the instability, fluctuations in flame surface at a large radial distance also result in larger heat release fluctuations. If the appropriate phase difference occurs between the heat release and pressure fluctuations, large surface area oscillations at large radial distances can strengthen and amplify the instability even further. 


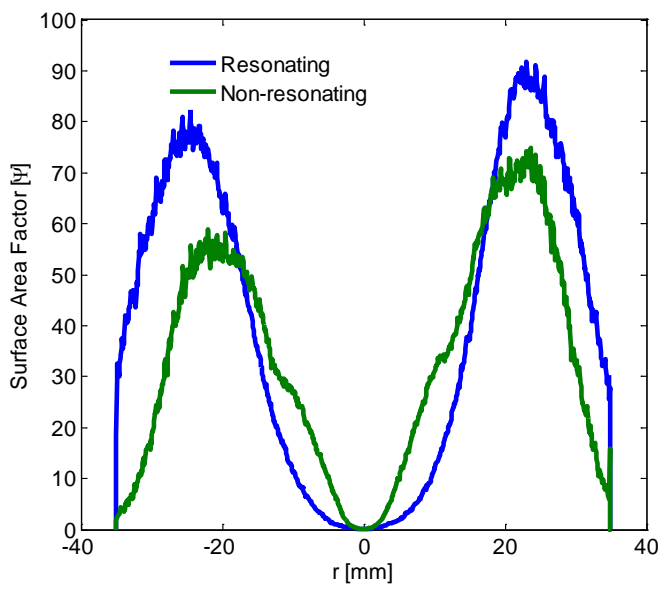

Figure 13. Radial distributions of flame surface area parameter, $\Psi$ (defined by Eq. 3 ) depicting the distribution of flame surface transversely across the burner.

If the flame is approximated as an inverted cone, the flame length would represent the length of the slanted surface. Two cones can have the same length of slanted side, but will have very different flame surface areas if the flame cone angle is large. This model represents the difference between the two cases. Both flames have roughly the same length, but the resonating flame has a larger cone angle, which distributes surface further away from the centerline. For resonating flames, the average area across all images is $369 \pm 128 \mathrm{~cm}^{2}$ and for non-resonating flames, the average is $305 \pm 66 \mathrm{~cm}^{2}$. The resonating flame displays a larger area by $20 \%$ and the range of fluctuations is larger.

\section{Time Variations of the Flame Surface}

If flame surface density (i.e., local heat release rate) is a direct cause of the combustion instability, then it would be expected that FSD should oscillate at nearly the same frequency as the pressure oscillations. To assess this idea the $\mathrm{kHz}$ PLIF data was interrogated and the results are listed in Tables 2-4. For the resonating case the FSD oscillates at $320 \mathrm{~Hz}$, as seen spectrum of FSD in Figure 14b. This result dictates that the flame surface area is modulated at the observed pressure fluctuation frequency and that the heat release will also oscillate at the acoustic frequency. Similar behavior was observed from complementary high-speed OH PLIF results by Steinberg et al. ${ }^{27}$. For the non-resonant case, the pressure fluctuations are negligible, however the FSD fluctuates at $10 \mathrm{~Hz}$ as seen in the spectrum of Figure 14a. This can be described as a slow breathing mode which scales with the radial velocity fluctuations and the diameter of the GTMC.

(a)

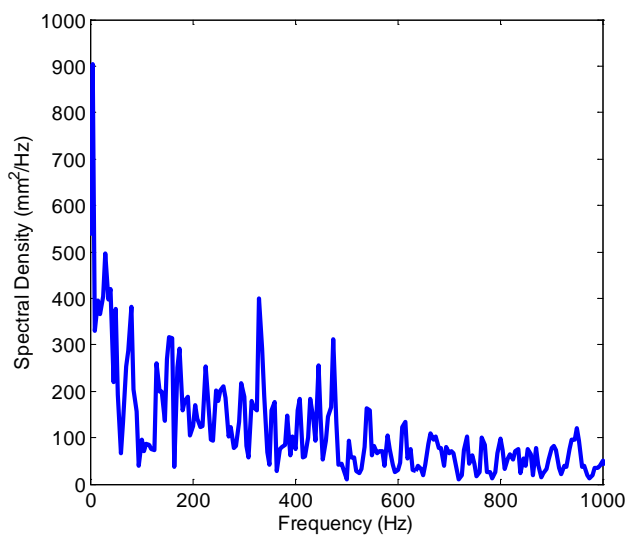

(b)

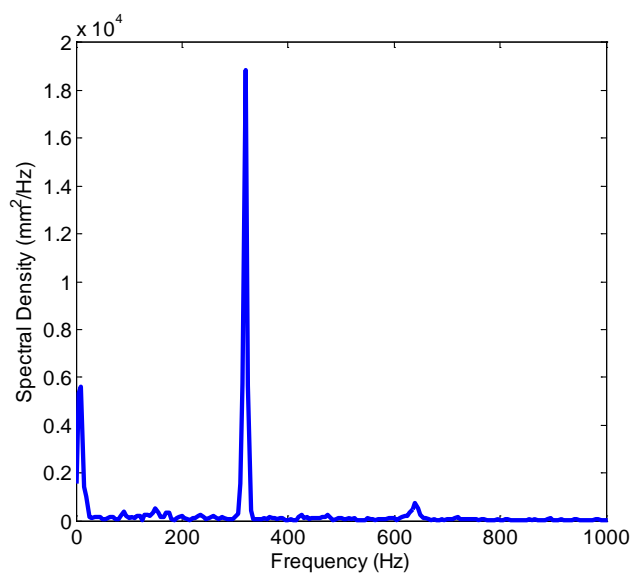

Figure 14. Spectra of flame surface density from kHz PLIF images. (a) Non-resonating flame showing a peak at $10 \mathrm{~Hz} \mathrm{~Hz}$. (b) Resonating flame, showing much larger peaks at $10 \mathrm{~Hz}$ and $320 \mathrm{~Hz}$. The $320 \mathrm{~Hz}$ peak corresponds to the frequency of pressure oscillations and $10 \mathrm{~Hz}$ is an asymmetric breathing mode. 
From the high-speed videos of the motion of the formaldehyde scalar field, it is apparent that there is a lateral variation in time of increased FSD from one lobe to another. If each lobe is considered as an independent region of interest, the time evolution can be considered which will isolate asymmetric behavior in the flame surface, which is lost when considering the combined motions of both lobes within the entire field of view. For resonant flames, the total integrated FSD in each lobe independently oscillates at the acoustic frequency. However, in the non-resonant case, there is a strong oscillation observed at $475 \mathrm{~Hz}$. Although, not directly measured in this study, measurements taken at DLR Stuttgart by Boxx et al. ${ }^{28}$ have contributed motions near this frequency to be caused by the rotation of the PVC.

The relative time-resolved behavior of each lobe can be considered by evaluating the ratio of the total flame surface signal in each lobe. The relative lobe motion captures the in-plane change in FSD across the centerline as affected by the PVC motion. For the non-resonant flame, the signal from one lobe to the other fluctuates at $475 \mathrm{~Hz}$, corresponding to the PVC. However, in the resonating flame, the ratio of the signal oscillates at $525 \mathrm{~Hz}$. Again, it is hypothesized that this oscillation corresponds to the rotation of the PVC through the plane of the laser sheet. From the time history of the signal in each lobe, it was observed that there is a phase shift of $127^{\circ}$ in non-resonant flames and a shorter phase shift of $89^{\circ}$ in resonant flames corresponding to the relative faster rotation of the PVC. In contrast to the total FSD spectra in Figure 14, the relative lobe motion spectra exhibit strong single peaks at the hypothesized PVC rotation frequency.

The time-resolved behaviors observed in this case were captured with the ability to better identify spatial variations in flame with the use of high-speed PLIF. For both cases, large radial variations in FSD were observed and there appears to be influences of the PVC on the motion of the flame surface. This behavior is asymmetric due to the helical structure of the PVC and would not be captured in line-of-sight chemiluminescence images.

\section{Flame Surface Response to Parameter Variations}

The equivalence ratio and air mass flow rate was varied to investigate changes to the flame surface structure and motion. Varying trends were observed for a combination of cases which resulted in resonating, weakly resonating, non-resonating, and highly lifted V-flames. This distribution of behaviors allowed for observation of the effect of instability processes and PVC interactions.

Air mass flow rate variation was conducted at two constant equivalence ratios, $\varphi=1$ and 1.2. The air flow rate was varied from 170 to $282 \mathrm{~g} / \mathrm{min}$. Decreasing the flow rate tended to reduce the instability strength. The combination of low air flow and high equivalence ratio produced a highly lifted, non-resonating DME flame. Tables 2 and 3 represent the results of this parameter study. It appears that for weakly resonating flames, the observed frequencies are due to both acoustic and flowfield oscillations. When the instability is weak, it cannot damp out the flowfield influence via the PVC. The weakly resonating low flow rate case at $\varphi=1$ exhibited an average structure similar to the non-resonating flames, with two zones in each lobe.

With increasing flow rate, the phase shift between the switching lobes is decreased and the surface area and RMS area fluctuations increase. The instability becomes stronger with a highly wrinkled flame which fluctuates strongly. The frequency of the lobe ratio exhibits the PVC frequency except in the low flow cases, where it is suspected that there is no PVC present. Not all swirling flowfields can support a PVC. As well, the detected $570 \mathrm{~Hz}$ is a harmonic of the $285 \mathrm{~Hz}$ observed in the ratio of the lobe signals. Therefore, the $570 \mathrm{~Hz}$ may not necessarily be due to a flowfield structure motion at that frequency.

In general, as the air mass flow rate is increased the acoustic and PVC frequencies also increase. As well, the flame lengths between resonating and non-resonating flames are all about the same. However, the degree of fluctuations, given by the RMS, increases for resonating flames. This is also true of the mean surface area and its RMS. Resonating flames have higher degrees of wrinkling and flame surface area, and these metric also oscillate to a greater extent than in quiet flames. 
Table 2. Flame surface response to the variation of air mass flow rate at a constant $\varphi=1$.

\begin{tabular}{cccc}
\hline Fuel Type & DME & DME & DME \\
Air [g/min] & 170 & 210 & 282 \\
Phi & 1 & 1 & 1 \\
\hline Instability Present & Weak & Weak & Yes \\
Acoustic Frequency [Hz] & 280 & 280 & 310 \\
FSD Frequency [Hz] & $270 / 570$ & 280 & 310 \\
Total Formaldehyde Frequency [Hz] & $270 / 570$ & 280 & 310 \\
Individual Lobe Frequency [Hz] & 280 & $280 / 345$ & 310 \\
Lobe Ratio Frequency [Hz] & 285 & 345 & 480 \\
Two- Zone Relative Frequency [Hz] & 285 & $\mathrm{~N} / \mathrm{A}$ & $\mathrm{N} / \mathrm{A}$ \\
Two-Zone Phase Shift [deg] & 75 & $\mathrm{~N} / \mathrm{A}$ & $\mathrm{N} / \mathrm{A}$ \\
Relative Lobe Phase Shift [deg] & 110 & 98 & 72 \\
Flame Surface Area Mean [ [m $^{2}$ ] & 210 & 191 & 326 \\
Flame Surface Area RMS [cm ${ }^{2}$ ] & 54 & 65 & 118 \\
Flame Length Mean [mm] & 100 & 81 & 103 \\
Flame Length RMS [mm] & 24 & 25 & 41.5 \\
\hline
\end{tabular}

Table 3. Flame surface response to the variation of air mass flow rate at a constant $\varphi=1.2$.

\begin{tabular}{cccc}
\hline Fuel Type & DME & DME & DME \\
Air [g/min] & 170 & 210 & 282 \\
Phi & 1.2 & 1.2 & 1.2 \\
\hline Instability Present & Highly Lifted & Yes & Yes \\
Acoustic Frequency [Hz] & 15 & 290 & 320 \\
FSD Frequency [Hz] & 5 & 290 & 320 \\
Total Formaldehyde Frequency [Hz] & 15 & 290 & 320 \\
Individual Lobe Frequency [Hz] & 5 & 290 & 320 \\
Lobe Ratio Frequency [Hz] & 20 & 379 & 525 \\
Two- Zone Relative Frequency [Hz] & $\mathrm{N} / \mathrm{A}$ & $\mathrm{N} / \mathrm{A}$ & $\mathrm{N} / \mathrm{A}$ \\
Two-Zone Phase Shift [deg] & $\mathrm{N} / \mathrm{A}$ & $\mathrm{N} / \mathrm{A}$ & $\mathrm{N} / \mathrm{A}$ \\
Relative Lobe Phase Shift [deg] & 61 & 89 & 89 \\
Flame Surface Area Mean [cm $\left.{ }^{2}\right]$ & 85 & 319 & 369 \\
Flame Surface Area RMS [cm $\left.{ }^{2}\right]$ & 42 & 107 & 128 \\
Flame Length Mean [mm] & 42 & 118 & 135 \\
Flame Length RMS [mm] & 17 & 35 & 48 \\
\hline
\end{tabular}

A special case to this investigation was the highly lifted flame. This case may be due to poor mixing as a result of decreased shear and recirculation at low flow rates, combined with increased fueling. Essentially the flame mixture does not become flammable until further downstream. This flame only experiences slow, breathing type oscillations which may be due to thermo-diffusive instabilities in the flame surface. As well, this flame is much less wrinkled than all other flames studied.

Variation of the equivalence ratio yielded similar trends to the variation of air mass flow rate. Thus some of these trends may just be related to general increase in the thermal power of the flame. In general the acoustic frequency (if present) and the PVC frequencies shift higher with more fueling. As well, when the flame transitions 
to a resonating state, the phase shift between the lobe oscillations also decreases. Finally, the total surface area increases with higher equivalence ratio, and the RMS of the area and length fluctuations is increased as well, as seen in Table 4.

These variations show that between loud, semi-loud, and quiet flames, there are competing effects for what dominates the flame surface motion. For resonating cases, the instability controls the flowfield and damps out effects due to the PVC. For non-resonating flames, the turbulent structures like the PVC and vortices greatly influence the oscillatory pattern of the flame. However, for weakly resonating flames, the two forces compete and balance is achieved in that the effects of both are observed.

Table 4. Flame surface response to the variation of equivalence ratio at constant air mass flow rate.

\begin{tabular}{cccc}
\hline Fuel Type & DME & DME & DME \\
Air [g/min] & 282 & 282 & 282 \\
Phi & 0.75 & 1 & 1.2 \\
\hline Instability Present & No & Yes & Yes \\
Acoustic Frequency [Hz] & N/A & 310 & 320 \\
FSD Frequency [Hz] & 10 & 310 & 320 \\
Total Formaldehyde Frequency [Hz] & 50 & 310 & 320 \\
Individual Lobe Frequency [Hz] & 475 & 310 & 320 \\
Lobe Ratio Frequency [Hz] & 475 & 480 & 525 \\
Two- Zone Relative Frequency [Hz] & 475 & $\mathrm{~N} / \mathrm{A}$ & $\mathrm{N} / \mathrm{A}$ \\
Two-Zone Phase Shift [deg] & 75 & $\mathrm{~N} / \mathrm{A}$ & $\mathrm{N} / \mathrm{A}$ \\
Relative Lobe Phase Shift [deg] & 127 & 72 & 89 \\
Flame Surface Area Mean [cm $\left.{ }^{2}\right]$ & 305 & 326 & 369 \\
Flame Surface Area RMS [cm ${ }^{2}$ ] & 65.6 & 118 & 128 \\
Flame Length Mean [mm] & 143 & 103 & 135 \\
Flame Length RMS [mm] & 28.2 & 41.5 & 48 \\
\hline
\end{tabular}

\section{Conclusion}

A gas turbine model combustor was found to exhibit a natural combustion instability that creates a pressure spectrum that has a single peak. The frequency of the instability increases with gas velocity, and this indicates the presence of an acoustic-convective mode. Models of such instabilities show that the convection time scale determines the amplitude and frequency of the instability. It was observed that flame shape plays an important role; flat flames exhibit a strong instability whereas V-shaped flames are typically quiet. For flat flames, the entire flame has the same convective time scale (equal to the distance from burner to flame / flow axial velocity). This convection time scale varies along a V-flame, so only a small segment of the flame can amplify the pressure oscillations. Flame geometry in this GTMC is controlled by mixing and the upstream flow pattern; specifically the amount of radial velocity imparted by the distribution of flow through each swirler.

Dimethyl ether flames exhibit similar instability behaviors as compared to other commonly studied fuels. The acoustic characterization of DME has shown that the frequency of the instability generally resonates in the range of 280-360 Hz. The pressure field, chemiluminescence, and flame front motion all oscillate at the same frequency; indicative of coupling between pressure, heat release, and velocity fluctuations. Rayleigh indices corroborate the observation that there is some degree of coupling between the heat release and pressure field in the GTMC. Although the coupling does not appear to be as strong as other fuels, the flat flame shape achieved with DME is consistent with conditions that foster combustion instabilities.

It is shown that flame geometry, including its spatial and temporal variations, can be more accurately determined using $\mathrm{kHz}$ PLIF than by conventional chemiluminescence. Spatial variations of flame surface density 
(and heat release rate) in resonating flames differed from that of non-resonating flames. The resonating case has larger flame surface area and peak FSD was located at larger radial distances than for the non-resonating case. When the instability is present the magnitude of area fluctuations was much greater, indicating large scale surface creation and destruction. The combination of large surface area and large area fluctuations leads to increased amplification of the instability.

The strength of high-speed PLIF was observed in the analysis of the time evolution of the flame surface structure with the spectrum of flame surface area oscillations. Surface area and thus heat release rate were seen to oscillate at the same $320 \mathrm{~Hz}$ frequency as pressure fluctuations. This coupling of pressure and heat release oscillations leads to amplification of the instability based on the Rayleigh criterion. However, the flame surface also exhibited asymmetric motions at $525 \mathrm{~Hz}$ associated with the presence of the PVC. In non-resonant flames, oscillations in flame surface density also occur at a low-frequency $(10 \mathrm{~Hz})$ due to a breathing mode and at the PVC rotation rate $(475 \mathrm{~Hz})$.

\section{Acknowledgments}

This research was funded by ONR under grant N00014-10-10561 and by the DOE-UTSR program under grant FE0007060. The authors thank Dr. Wolfgang Meier of DLR Stuttgart for permission to use the GTMC design.

\section{References}

[1] Gupta, A.K., Lilley, D.G., and Syred, N., Swirl Flows, Abacus Press, Kent, 1984.

[2] Rayleigh, J.W.S. and Lindsay, R., The Theory of Sound: Vol. 2, Dover Publications, 1945, p. 230.

[3] Lieuwen, T., Torres, H., Johnson, and C., Zinn, B.T., "A mechanism of combustion instability in lean premixed gas turbine combustors”, J. Eng. Gas Turb. Power. 123: 182-189 (2001).

[4] Allison, P.M., Driscoll, J.F., and Ihme, M., "Acoustic characterization of a partially-premixed gas turbine model combustor: syngas and hydrocarbon fuel comparisons”, Proc. Combust. Inst. 34: 3145-3153 (2013).

[5] Giezendanner, R., Weigand P., Duan X.R., Meier W., Meier, U., and Aigner M., "Laser-based investigations of periodic combustion instabilities in a gas turbine model combustor”, J. Eng. Gas Turb. Power, 127: 492-496 (2005). [6] Weigand P., Meier W., Duan X.R., Stricker W., and Aigner M., "Investigations of swirl flames in a gas turbine model combustor I. Flow field, structures, temperature, and species distributions”, Combust. Flame, 144: 205-224 (2006).

[7] Allison, P.M., Rosenberg, D.A., and Driscoll, J.F., "Combustion instability interaction with fuel-air mixing in a partially-premixed gas turbine model combustor," $8^{\text {th }}$ US National Combustion Meeting, Park City, Utah, May 2013. [8] Duan X.R., Meier W., Weigand P., and Lehmann, B., "Phase-resolved laser Raman scattering and laser Doppler velocimetry applied to periodic instabilities in a gas turbine model combustor", Applied Physics B: Lasers and Optics, 80: 389-396 (2005).

[9] Andersson, Ö., Neij, H., and Bood, J., "Optical Characterization of Dimethyl Ether (DME) for Laser-based Combustion Diagnostics,” Combustion Science and Technology, vol. 137, 1998, pp. 299-322.

[10] Fischer, S., Dryer, F., and Curran, H., "The Reaction Kinetics of Dimethyl Ether, I: High Temperature Pyrolysis and Oxidation in Flow Reactors,” Journal of Chemical Kinetics, 2000.

[11] Curran, H., Fischer, S., and Dryer, F., "The Reaction Kinetics of Dimethyl Ether, II: Low Temperature Oxidation in Flow Reactors,” Journal of Chemical Kinetics, 2000.

[12] Mcilroy, A., Hain, T. D., Michelsen, H. a., and Cool, T. a., "A laser and molecular beam mass spectrometer study of low-pressure dimethyl ether flames," Proceedings of the Combustion Institute, vol. 28, Jan. 2000, pp. 16471653.

[13] Kaiser, E. W., Wallington, T. J., Hurley, M. D., Platz, J., Curran, H. J., Pitz, W. J., and Westbrook, C. K., "Experimental and Modeling Study of Premixed Atmospheric-Pressure Dimethyl Ether-Air Flames," The Journal of Physical Chemistry A, vol. 104, Sep. 2000, pp. 8194-8206.

[14] Zhao, Z., Kazakov, a., and Dryer, F. L., "Measurements of dimethyl ether/air mixture burning velocities by using particle image velocimetry,” Combustion and Flame, vol. 139, Oct. 2004, pp. 52-60.

[15] Qin, X., and Ju, Y., "Measurements of burning velocities of dimethyl ether and air premixed flames at elevated pressures," Proceedings of the Combustion Institute, vol. 30, Jan. 2005, pp. 233-240.

[16] Brackmann, C., Bood, J., Aldén, M., Pengloan, G., and Andersson, Ö., "Quantitative Measurements of Species and Temperature in a DME-Air Counterflow Diffusion Flame Using Laser Diagnostic Methods," Combustion Science and Technology, vol. 178, Jun. 2006, pp. 1165-1184. 
[17] Wang, Y. L., Holley, A. T., Ji, C., Egolfopoulos, F. N., Tsotsis, T. T., and Curran, H. J., "Propagation and extinction of premixed dimethyl-ether/air flames," Proceedings of the Combustion Institute, vol. 32, Jan. 2009, pp. 1035-1042.

[18] Fuest, F., Barlow, R. S., Chen, J.-Y., and Dreizler, A., "Raman/Rayleigh scattering and CO-LIF measurements in laminar and turbulent jet flames of dimethyl ether," Combustion and Flame, vol. 159, Aug. 2012, pp. 2533-2562. [19] Gabet, K. N., Shen, H., Patton, R. A., Fuest, F., and Sutton, J. A., "A comparison of turbulent dimethyl ether and methane non-premixed flame structure,” Proceedings of the Combustion Institute, vol. 34, Jan. 2013, pp. 14471454.

[20] Sadanandan, R., Stöhr, M., and Meier, W., "Flowfield-flame structure interactions in an oscillating swirl flame”, Combustion, Explosion, and Shock Waves 45: 518-529 (2009).

[21] Steinberg, A., Boxx, I., Stöhr, M., Meier, W., and Carter, C., "Effects of flow structure dynamics on thermoacoustic instabilities in swirl-stabilized combustion”, AIAA J. 50 (4): 952- 967 (2012).

[22] Pun, W., Palm, S.L., and Culick, F.E.C., "Combustion dynamics of an acoustically forced flame”, Combust. Sci. and Tech. 175: 499-521 (2003).

[23] Kang, D.M., Culick, F.E.C., and Ratner, A., “Combustion dynamics of a low-swirl combustor”, Combust. Flame 151: 412-425 (2007).

[24] Tap, F.A., Hilbert, R., Thevenin, D., and Veynante, D., “A generalized flame surface density modelling approach for the auto-ignition of a turbulent non-premixed system”, Combust. Theory Modelling 8:165-193 (2004).

[25] Boger, M., Veynante, D., Boughanem, H., and Trouve, A., "Direct numerical simulation analysis of flame surface density concept for large eddy simulation of turbulent premixed combustion", Proc. Combust. Institute 27: 917-925 (1998).

[26] Kirkpatrick, M.P., Armfield, S.W., Masri, A.R., and Ibrahim, S.S., "Large eddy simulation of a propagating turbulent premixed flame”, Flow, Turb., Combust. 70: 1-19 (2003).

[27] Steinberg, A.M., Boxx, I., Stöhr, M., Carter, C.D., and Meier, W., "Flow-flame interactions causing acoustically coupled heat release fluctuations in a thermo-acoustically unstable gas turbine model combustor", Combust. Flame 157: 2250-2666 (2010).

[28] Boxx, I., Stöhr, M., Carter, C., and Meier, W., "Temporally resolved planar measurements of transient phenomena in a partially pre-mixed swirl flame in a gas turbine model combustor”, Combust. Flame 157: 15101525 (2010). 